\title{
Design of a Test Setup to Measure Magnetic Signature Reduction
}

\author{
Djurre Wikkerink, Armando Rodrigo Mor, Henk Polinder, Robert Ross \\ Delft University of Technology \\ Delft, The Netherlands \\ d.p.wikkerink@tudelft.nl
}

\section{Synopsis}

In order to avoid detection by sea mines, the magnetic signature of merchant and naval vessels can be reduced by running a current through a set of on-board copper coils. This process is called degaussing. Studies have shown that the volume, weight and energy losses of a degaussing system can be reduced by replacing the copper coils with high temperature superconductive (HTS) coils. Moreover, since the technology and production of HTS has matured and the material is highly available, the use of HTS for degaussing coils is a serious option. As a preliminary study towards an HTS degaussing test setup, this paper presents the design of a table-top demonstration with copper degaussing coils. The goal of the demonstration is to measure the magnetic signature and the magnetic signature reduction of a cylindrical object. The design choices of the test setup and the measuring system are discussed. The magnetic signature of the table-top model is calculated as well as the optimal placement of the degaussing coils and the optimal degaussing currents. These results are compared with measurements of the magnetic flux density around the demonstrator.

Keywords - degaussing, deperming, magnetic signature, high temperature superconductor, magnetic field measurements.

\section{Introduction}

Due to their permeability, steel ships distort Earth's magnetic field. These distortions in the magnetic field around the vessel can be detected by sensors attached to sea mines. In order to reduce the risk of a mine activation, it is necessary to reduce the magnetic signature. This can either be done passively, by using non-ferrous material for the hull, or actively by degaussing systems. The magnetic signature of a ship consists of various components (Holmes 2006). The total magnetic signature, $\boldsymbol{B}_{\text {sig }}$, can be expressed as a sum of the components:

$$
\boldsymbol{B}_{\text {sig }}=\boldsymbol{B}_{\text {ind }}+\boldsymbol{B}_{\text {perm }}+\boldsymbol{B}_{e d d y}+\boldsymbol{B}_{c o r}+\boldsymbol{B}_{e q}
$$

where $\boldsymbol{B}_{\text {ind }}$ is the magnetic field induced by Earth's magnetic field, $\boldsymbol{B}_{\text {perm }}$ is the permanent magnetisation of the hull due to construction or long exposure to an external field, $\boldsymbol{B}_{\text {eddy }}$ is the magnetic field which is induced by eddy currents in the hull due to movement in Earth's magnetic field, $\boldsymbol{B}_{\text {cor, }}$ is the magnetic field induced by corrosion currents and $\boldsymbol{B}_{e q}$ is the magnetic field due to on-board equipment, like generators or converters. The magnetic signature can be reduced actively by a set of on-board degaussing coils. These coils create an opposing magnetic field to the induced magnetic field, so that the net magnetic field is zero. Degaussing coils can either be placed in longitudinal direction (L-coils), athwart ship (Acoils) or in the vertical direction of the ship (Mcoils). The process of degaussing can be expressed as follows:

$$
\boldsymbol{B}=\boldsymbol{B}_{\text {Earth }}+\boldsymbol{B}_{\text {sig }}-\boldsymbol{B}_{\text {coils }}
$$

where $\boldsymbol{B}$ is the magnetic field which is measured around the ship, $\boldsymbol{B}_{\text {Earth }}$ is Earth's magnetic field and $\boldsymbol{B}_{\text {coils }}$ is the magnetic field produced by the degaussing coils which, ideally, equals the magnetic signature, $\boldsymbol{B}_{\text {sig. }}$.

Nowadays, degaussing coils are made of copper. This results in a bulky system with heavy coils and a significant amount of heat loss. The performance of a degaussing system is expected to improve by replacing the copper coils with high temperature superconductive (HTS) coils (Ross 2012). Experimental work has already been done by replacing one of the degaussing coils on a naval ship with an HTS coil (Kephart 2011). HTS coils can have a larger current density, so less material for the coils is needed. Also, the resistance of a superconductive coil is nearly zero, so the Ohmic losses are negligible. A drawback, however, is that HTS only operates at cryogenic temperatures, so the system needs to be cooled with liquid nitrogen. 
In order to investigate and verify if the use HTS for degaussing is feasible, a table-top demonstration of HTS degaussing is planned in the near future.

As a preliminary research, this paper presents a test setup which is able to measure the magnetic signature and the magnetic signature reduction of a steel object with copper degaussing coils.

\section{System Desription}

\subsection{Design of the setup}

The table top demonstration consists of a steel hollow cylinder which is equiped with a set of three degaussing coils. The magnetic signature of the pipe and the effect of the degaussing coils can be measured with a movable sensor at a certain distance from the pipe along a line parallel to the longitudal direction of the pipe. Earth's magnetic field is the external field.

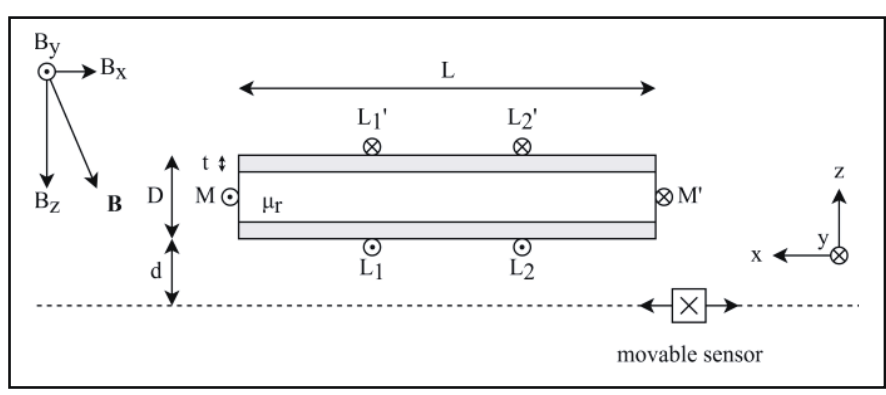

Figure 1: Schematic overview of the test setup

Figure 1 shows a schematic representation of the test setup where, $L$ is the length of the pipe, $D$ is the diameter of the pipe, $t$ is the thickness of the wall, $d$ the vertical distance of the sensor from the pipe and $\mu_{r}$ is the relative permeability of the material. $B_{x}, B_{y}$ and $B_{z}$ are the Cartesian components of Earth's magnetic field, with respect to reference frame $x, y$ and $z$. In this reference, $x$ is pointing to the magnetic south pole and $z$ is orthogonal to Earth's surface. $L_{1}, L_{2}$, and $M$ represent the three degaussing coils. The sensor can move over a length of 3 meters. Table 1 shows values for the parameters of the test setup.

Table 1: Parameters of the pipe

\begin{tabular}{|l|l|c|c|}
\hline \multicolumn{4}{|c|}{ Pipe parameters } \\
\hline Name & \multicolumn{1}{|c|}{ Description } & Value & Unit \\
\hline$L$ & pipe length & 1000 & $\mathrm{~mm}$ \\
\hline$D$ & pipe diameter & 200 & $\mathrm{~mm}$ \\
\hline$t$ & wall thickness & 10 & $\mathrm{~mm}$ \\
\hline$d$ & measuring distance & 190 & $\mathrm{~mm}$ \\
\hline$\mu_{r}$ & relative permeability & 110 & - \\
\hline$B_{x}$ & flux density in $x$ & -20.1 & $\mu \mathrm{T}$ \\
\hline$B_{y}$ & flux density in $y$ & 0.7 & $\mu \mathrm{T}$ \\
\hline$B_{z}$ & flux density in $z$ & -35.4 & $\mu \mathrm{T}$ \\
\hline
\end{tabular}

It should be noted that Earth's magnetic field was found not to be homogeneous at the measurement location. The values from table 1 are an average taken over the distance of the measuring line. Figure 2 shows a plot of the measured flux density.

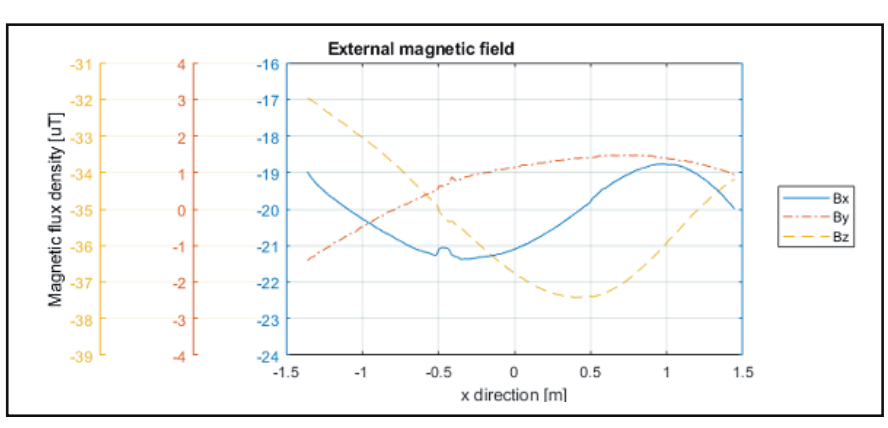

Figure 2: Measured background field

\subsection{Realization}

Figure 3 shows a picture of the the test setup which was built according to the specifications shown in table 1.

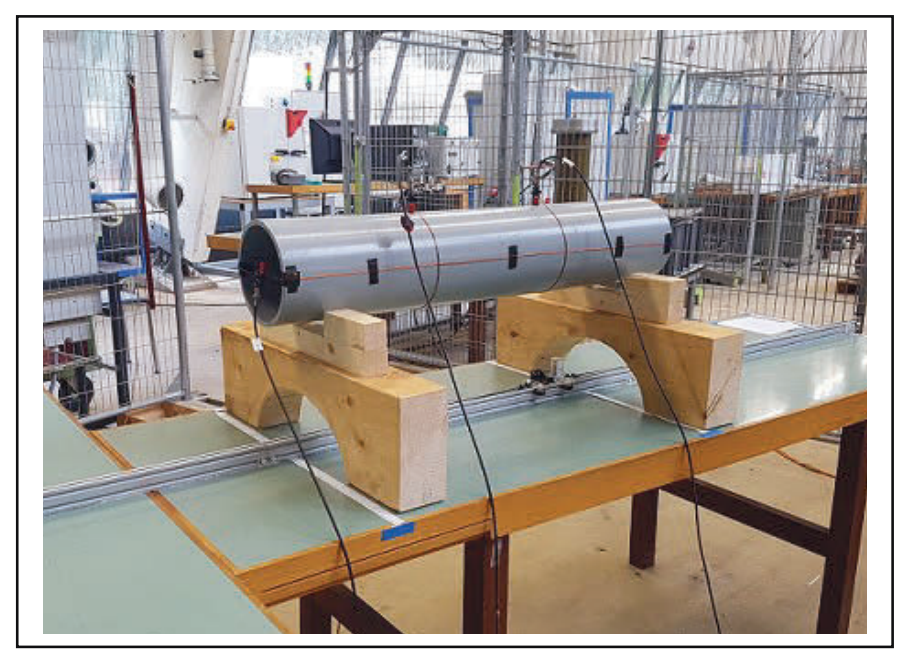

Figure 3: Picture of the test setup

The degaussing coils are wound around the pipe with regular copper wire. Each coil has a number of two turns. The coils are individually supplied by direct current power sources. The connection from the power source to the degaussing coils is through co-axial cables in order to minimize the influence of the magnetic field of the supply current on the measurements.

The material of the pipe is $\mathrm{S} 355 \mathrm{~J} 2 \mathrm{H}$; a high tensile steel type which is used for the construction of ships. Underneath the pipe, a rail is placed which transports the sensor. At the end of the rail, an encoder measures the position of the sensor. Figure 4 shows the rail with the sensor cart.

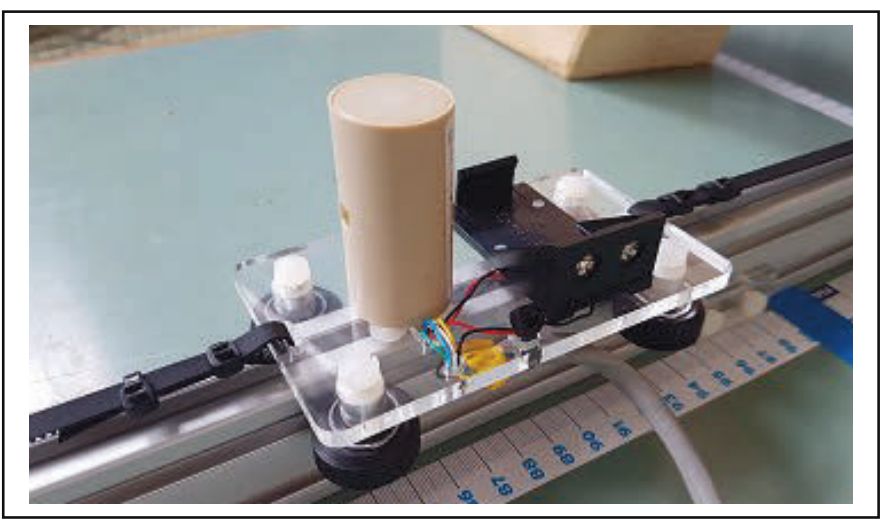

Figure 4: Movable sensor attached to the rail

The magnetic flux density underneath the pipe is measured with a fluxgate sensor (Stefan Mayer 
FLC3-70). Fluxgate sensors are known for their precision, however, the accuracy is relatively low. The fluxgate principle is based on magnetization of a soft iron core inside the sensor. Due to a small permanent magnetization of the core, an offset can occur. Therefore, all the measurements of the magnetic signature are calibrated to a reference measurement of the magnetic flux density in the room.

\subsection{Deperming}

During the fabrication process of the steel or the construction process of the pipe, the pipe might have been permanently magnetized. In order to get rid of any permanent magnetization within the pipe, it needs to be demagnetized. By applying a decreasing alternating magnetic flux density in the material, the permanent magnetization can be removed (Holmes 2008). Figure 5 shows a picture of a deperming coil around the test object.

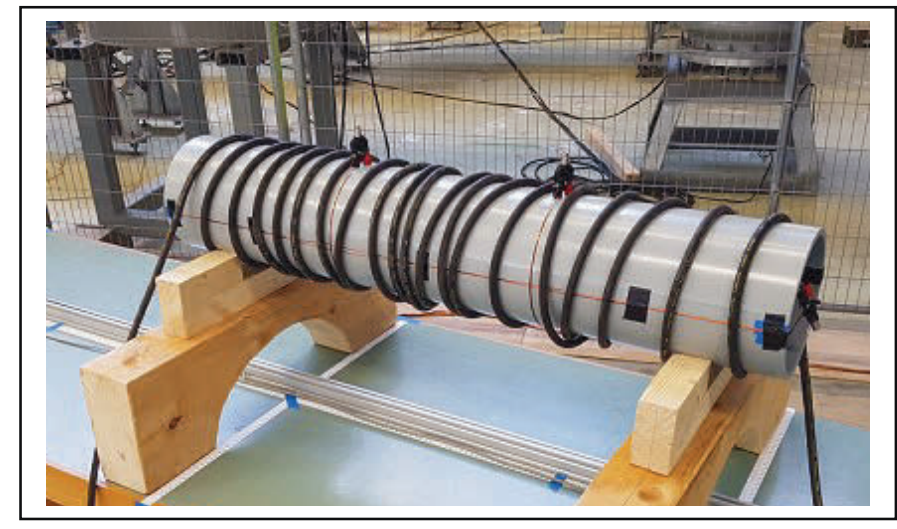

Figure 5: Deperming of the pipe

With an AC current through the coil starting at 15 Ampere, the magnetic flux density was slowly decreased to zero. The frequency of the current was 50 Hertz. Figure 6 shows the measured results of the magnetic signature of the pipe before and after the deperming procedure.

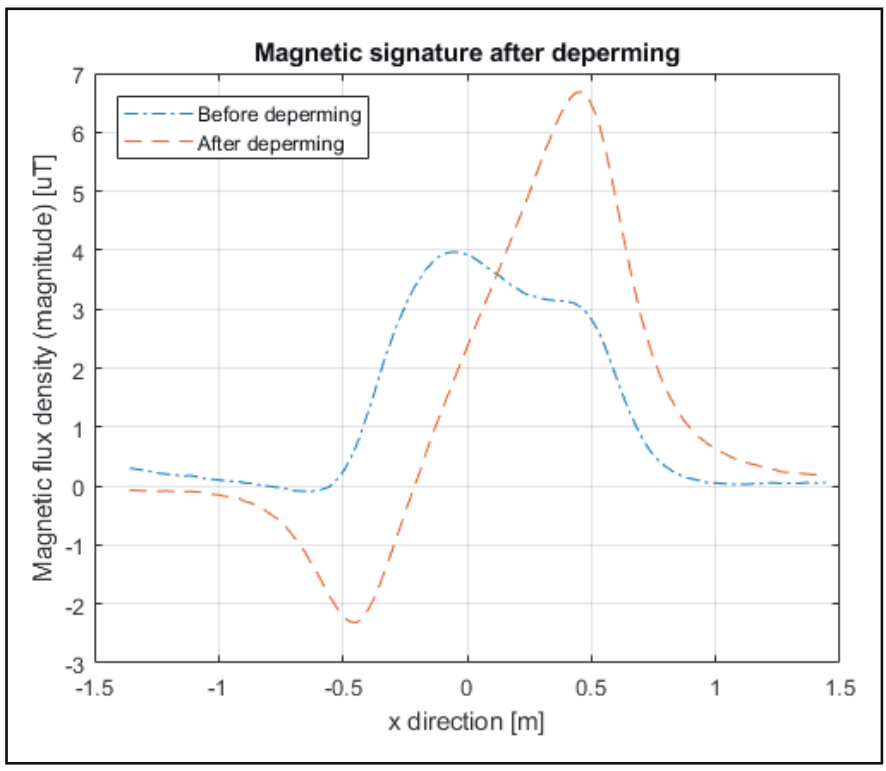

Figure 6: Magnetic signature before and after deperming

It can be seen from this graph that there was a significant permanent magnetization in the pipe.
The measured magnetic signature after deperming shows a high similarity with the simulated magnetic signature which is shown in the next section. This implies the deperming procedure was successful.

\section{Modelling}

In order to find the optimal placement for the Lcoils and the optimal amount of degaussing current, a model of the pipe is needed. This model should be usable in an optimization loop to optimize the coil placement and currents values.

\subsection{Magnetic field modelling}

There are various ways to model the magnetic field around a steel object. An analytic approach may be used when the object has a spherical or ellipsoidal geometry. This technique is based on solving Maxwell's equations in a ellipsoidal coordinate system (Baker 1982). However, the use of finite element modelling (FEM) shows more accurate results when an object has a different kind of geometry (Aird 2000). Figure 7 shows a graph where the results of analytical modelling using the ellipsiodal coordinate system are compared to a FEM approach. In the analytical model, the pipe is modelled as an ellipsoid which has the same length and width as the pipe. In the FEM model a CAD drawing of the pipe is used. The FEM modelling is done in COMSOL.

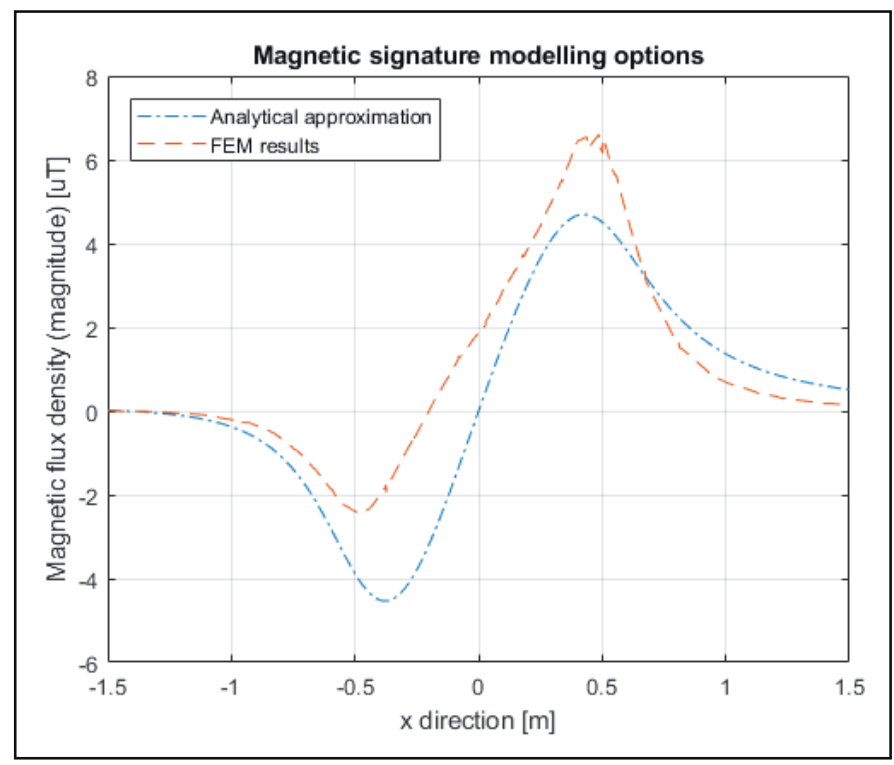

Figure 7: Analytical versus FEM results

Although the results do not completely match, the analytical results show some resamblance with the FEM results. The asymmetry in the plots is due to the incidence angle of the external field. The FEM results show a larger asymmetry than the analytic results which is due to the sharp edges of the pipe compared to the rounded edges of the ellipsoid. In this case, analytical modelling is useful to approximate the geometry of an object with a similar shape as an ellipsoid and to make a first estimation of the flux densities. It might also be 
used in optimization loops, because this technique requires less computing power than the use of FEM.

\subsection{Optimization procedure}

The goal of the optimization loop is to find the ideal coil placement and degaussing currents. This is done by minimizing the following optimization criterion, $c$ :

$$
c=\int\left(\frac{\boldsymbol{B}\left(I_{L 1}, I_{L 2}, I_{M 1}, x_{L 1}, x_{L 2}\right)}{\boldsymbol{B}_{d e s}}-1\right)^{2} d l
$$

where $l$ is placed on the measurement line, $\boldsymbol{B}$ is the magnetic flux density on the measurement line, which can be written as a function of optimization variables. These are the degaussing currents $I_{L 1}, I_{L 2}$ and $I_{M 1}$ and the L-coil positions $x_{L 1}$ and $x_{L 2}$. $\boldsymbol{B}_{d e s}$ is the desired magnetic flux density on the measurement line. For an optimal degaussing effect, the desired magnetic flux density on the measurement line should be as close to the flux density which would have been there if the pipe was not there, this is the case when $c$ is at a minimum. The solution for the optimal degaussing currents in Ampere-turns and the optimal placement of the L-coils are shown in table 2.

Table 2: Optimized optimization variables

\begin{tabular}{|l|l|c|c|}
\hline \multicolumn{4}{|c|}{ Parameters } \\
\hline Name & \multicolumn{1}{|c|}{ Description } & Value & Unit \\
\hline$I_{L 1}$ & current $L_{1}$ coil & 3.64 & $\mathrm{At}$ \\
\hline$I_{L 2}$ & current $\mathrm{L}_{2}$ coil & 3.64 & $\mathrm{At}$ \\
\hline$I_{M}$ & current $\mathrm{M}$ coil & 3.98 & $\mathrm{At}$ \\
\hline$x_{L 1}$ & position $\mathrm{L}_{1}$ coil & -167 & $\mathrm{~mm}$ \\
\hline$x_{L 2}$ & position $\mathrm{L}_{2}$ coil & 167 & $\mathrm{~mm}$ \\
\hline
\end{tabular}

In figure 8 , the expected magnetic signature in the $x z$ plane of the pipe is shown.

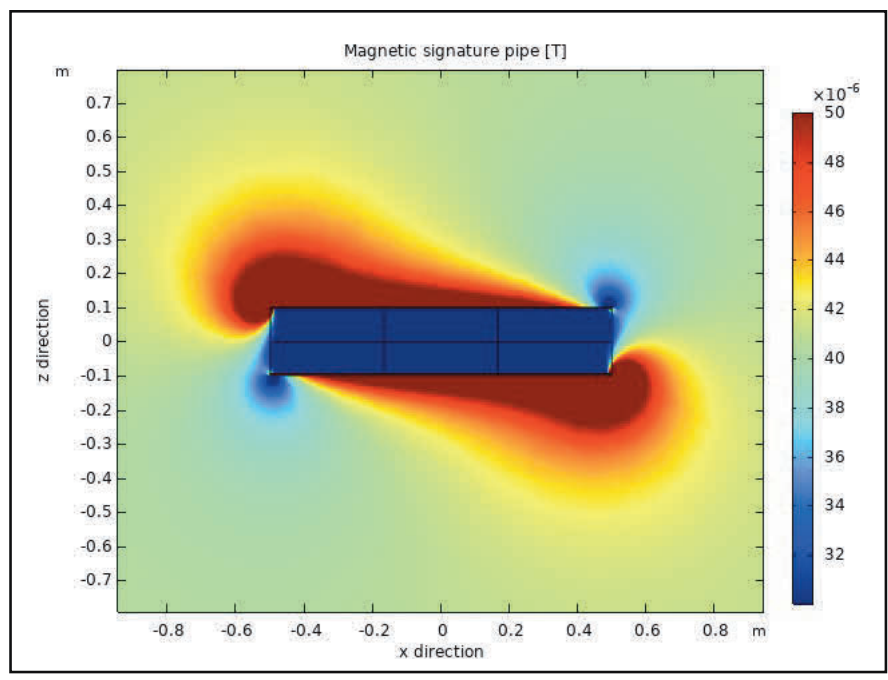

Figure 8: Simulated magnetic signature $x z$ plane

For comparison, the effect of the degaussing coils in the $x z$ plane is shown in figure 9 .

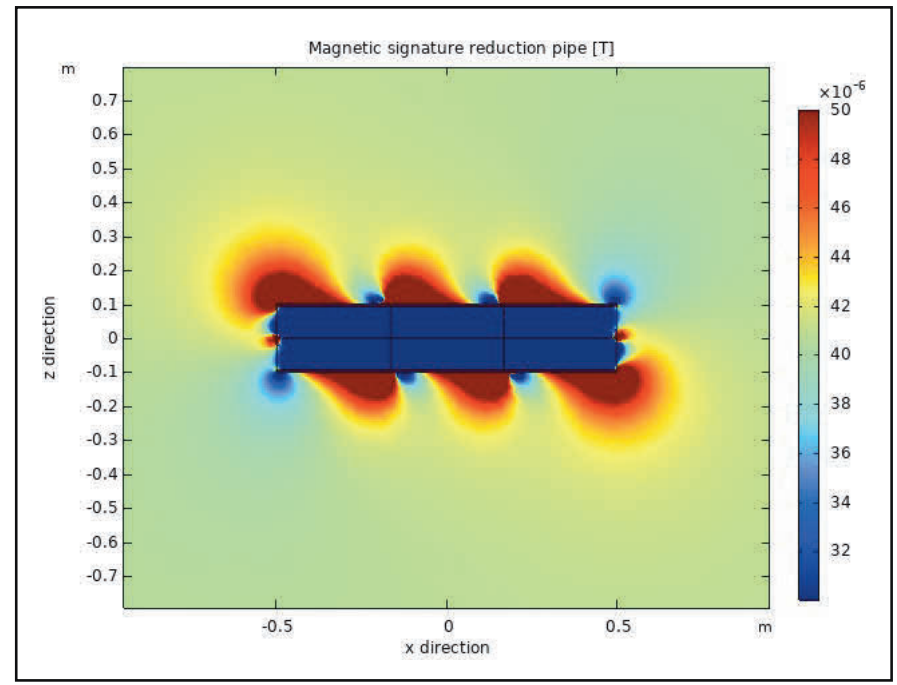

Figure 9: Simulated magnetic signature reduction in the $x z$ plane

\section{Results}

The measurements where done according to the following procedure. First, a reference measurement was done by mapping the magnetic flux density in the room without the presence of the pipe. Secondly, the magnetic signature of the pipe was measured by placing the pipe in its location. And finally, with the pipe in the same location, the magnetic signature reduction was measured by running the calculated degaussing currents through the degaussing coils. The magnetic signature and magnetic signature reduction measurements were normalized by subtracting the reference measurement. The magnetic flux denstiy is measured in the $x, y$ and $z$ direction. The $y$ direction is not shown here, because the external field only has an $x$ and $z$ component and the pipe is symmetrical in this plane.

In figure 10, the plots are shown of the measured and the simulated $x$-components (longitudinal) of the magnetic signature and the reduced magnetic signature.

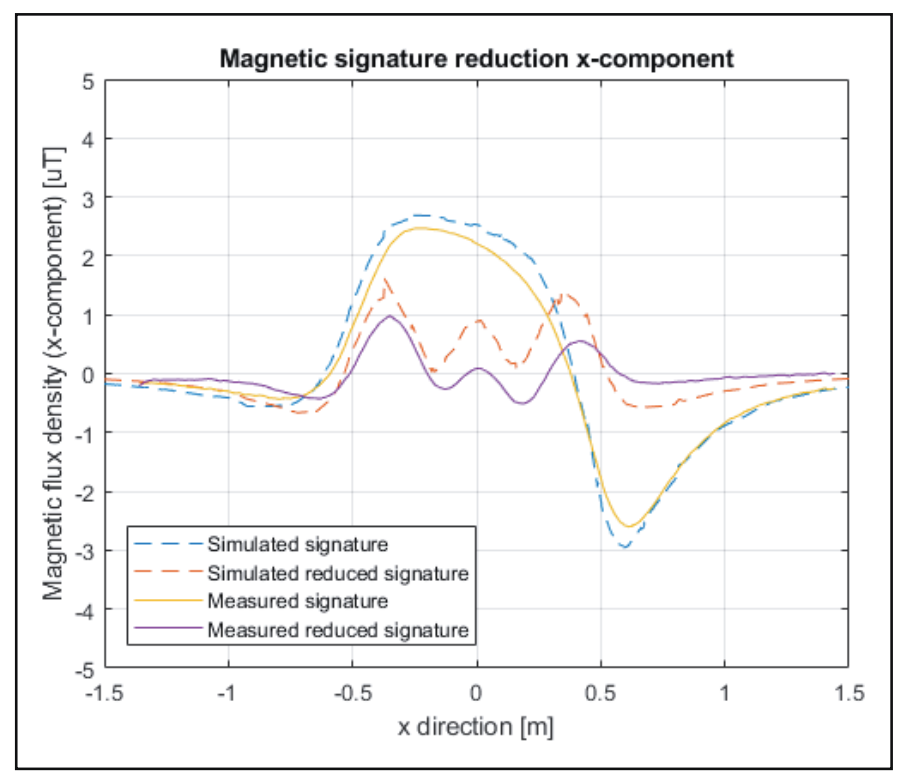

Figure 10: Magnetic signature reduction $x$ component 
Figure 11 shows the plots of the $z$-component (vertical) of the measured and simulated magnetic flux density.

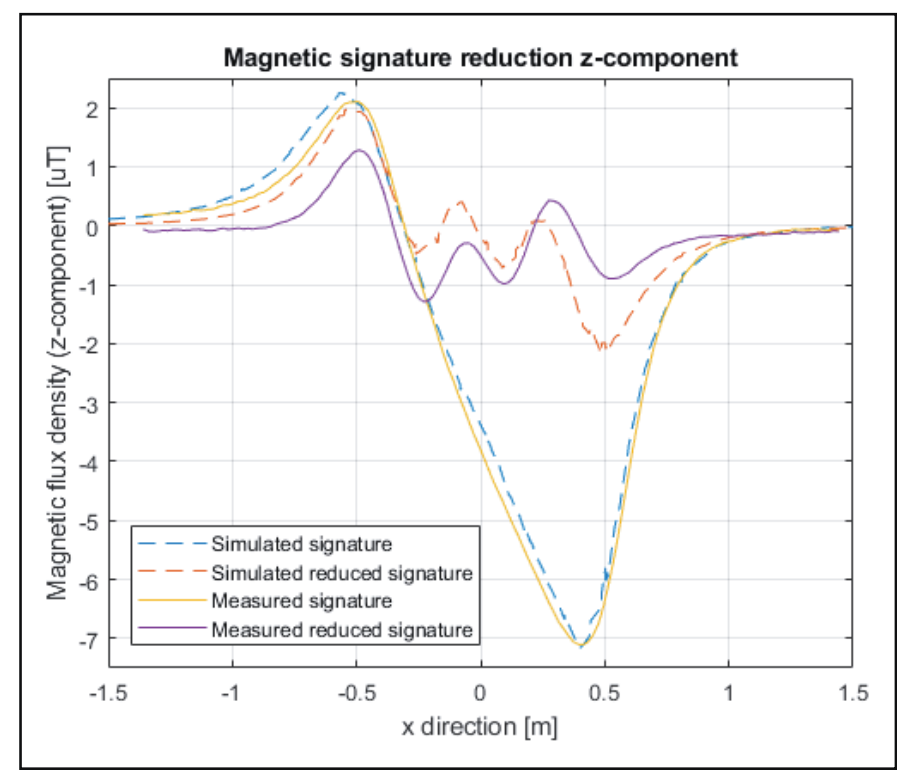

Figure 11: Magnetic signature reduction $z$ component

Finally, the plots of the magnitude of the measured magnetic flux density is shown in figure 12. Again, the measured results are compared with the simulated results.

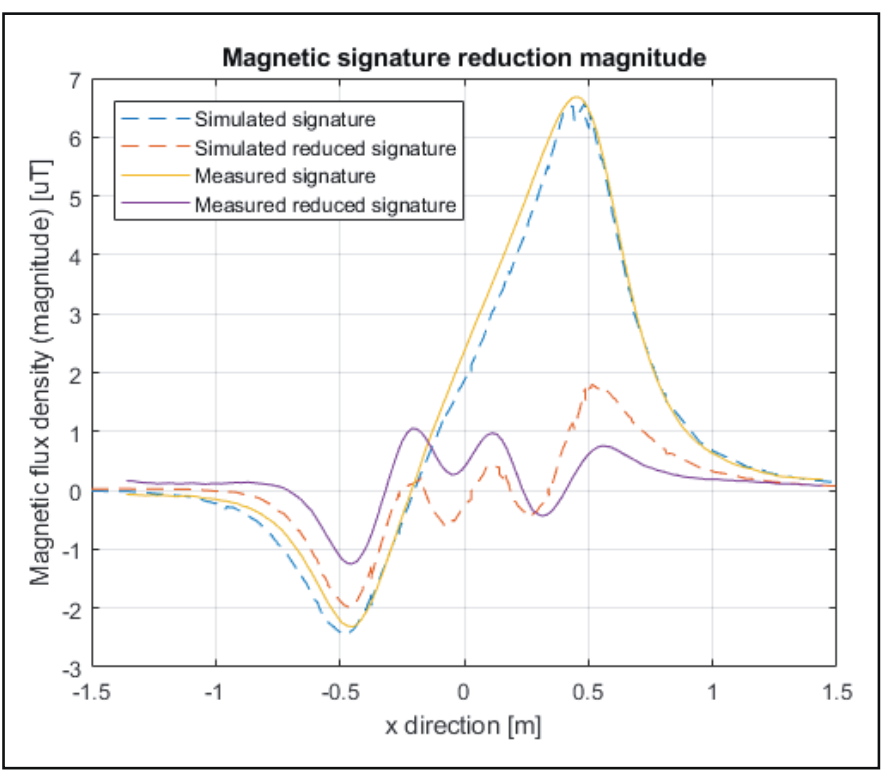

Figure 12: Magnetic signature reduction magnitude

It can be seen from the results that the measured magnetic signature is quite similar to the modelled magnetic signature, but, there is a small difference. The results of the measured magnetic signature reduction show a larger deviation from the simulated results.

\section{Discussion}

In the results, a difference is observed between the simulated and measured magnetic flux densities. One of the reasons is that the external magnetic flux density in the measuring room was not homogenous, while in the simulations an average of this magnetic flux density was used. Another reason could be that after the deperming process still some permanent magnetization remains in the pipe, which is not taken into account in the simulations. The simulated degaussing shows a larger deviation from measurements than the simulated magnetic signature. This might be due to the use of edge current modelling, which requires more investigation.

In future work a table-top model will be used with both copper and HTS coils. The goal is to not only compare static, but also dynamic behavior.

\section{Conclusion}

In this paper, a test setup is presented which can successfully measure the degaussing and deperming procedure of a steel pipe. The influence of the surrounding near the setup can influence and distort Earth's magnetic field in the nearby of the setup, which is an important factor in these kind of measurements. Removing the permanent magnetization of the test object is necessary, because its influence can be significant.

\section{References}

Aid GJC. 2000. Modelling the induced magnetic signature of naval vessels [dissertation]. Glasgow: University of Glasgow

Baker FE, Brown SH. 1982. Magnetic induction of ferromagnetic prolate spheroidal bodies and infinitesimally thin current bands. Journal of Appl. Physics. 53:3991-3996.

Holmes JJ. 2006. Exploitation of a ship's magnetic field signatures. [Place unknown]. Morgan \& Claypool.

Holmes JJ. 2008. Reduction of a ship's magnetic field. [Place unknown]. Morgan \& Claypool.

Kephart JT, Fitzpatrick BK, Ferrara P, Pyryt M, Pienkos J, Golda EM. 2011. High temperature superconducting degaussing from feasibility to fleet adoption. IEEE trans. appl. supercond. 21:2229-2232

Ross R, Meijer CG, van de Mheen RJ. 2012. Degaussing by normal and superconductive windings. Proc. $11^{\text {th }}$ INEC. May 15-17. Edinburgh. 